\title{
Síntesis de carveol, carvona, verbenol y verbenona
}

\author{
Rolando de J. Barrera*\$, Edwin A. Alarcón*, Lina M. González*, \\ Aída L. Villa*, Consuelo Montes de Correa* \\ * Grupo de Catálisis Ambiental, Departamento de Ingeniería Química, \\ Facultad de Ingeniería, Universidad de Antioquia, \\ Apartado Aéreo 1226, Medellín, Colombia \\ §rolando@udea.edu.co
}

(Recibido: Febrero 13 de 2008 - Aceptado: Abril 25 de 2008)

\begin{abstract}
Resumen
Se presenta una revisión sobre los principales sistemas catalíticos para la síntesis de carveol, carvona, verbenol y verbenona reportados entre 1990 y 2007. Estos compuestos se utilizan en la industria farmacéutica y de alimentos para la producción de fragancias y saborizantes. La creciente demanda de estos productos y la tendencia a nivel mundial de implementar procesos amigables con el ambiente hacen necesario mantener esfuerzos en la búsqueda de sistemas catalíticos de síntesis que resulten económica y técnicamente viables. Se hace énfasis en la oxidación alílica de limoneno y $\alpha$-pineno. Además, se presentan los avances más recientes de nuestro grupo de investigación en el estudio de reacciones catalíticas basadas en las ftalocianinas.
\end{abstract}

Palabras clave: Oxidación alílica, Limoneno, $\alpha$-pineno, Carveol, Carvona, Verbenol, Verbenona.

CHEMICAL ENGINEERING

\section{Synthesis of carveol, carvone, verbenol, and verbenone}

\begin{abstract}
This paper presents a review on catalytic systems, reported between 1990 and 2007, for the synthesis of carveol, carvone, verbenol, and verbenone. These products are used in the pharmaceutical and food industries as raw materials for the production of fragrances and flavors. The increasing demand for these products and the worldwide trend to implement environmentally-friendly processes makes it necessary to maintain efforts in the search for catalytic synthesis systems that turn out to be economically and technically feasible. Emphasis is made on the allylic oxidation of limonene and $\alpha$-pinene. Moreover, we present the most recent advances of our research group in the study of catalytic reactions based on phtalocyanines.
\end{abstract}

Keywords: Allylic oxidation, Limonene, $\alpha$-pinene, Carveol, Carvone, Verbenol, Verbenone. 


\section{Introducción}

El limoneno y el $\alpha$-pineno son monoterpenos provenientes de los aceites esenciales de naranja y trementina, respectivamente. La producción mundial del aceite esencial de naranja $(90 \%$ de limoneno) es de aproximadamente 50,000 ton (Duetz et al., 2000). Su precio de venta es de 1.5 US \$ / kg (Department of Primary Industries, 2002). La trementina se obtiene por exudación de árboles de pino (Coppen \& Hone, 1995), o como un subproducto de la industria papelera (Coppen \& Hone, 1995; Lindmark-Henriksson et al., 2003).

De la oxidación de monoterpenos se obtienen epóxidos, aldehídos, cetonas, ésteres y éteres, los cuales son intermediarios en la preparación de cosméticos, productos farmacéuticos, agroquímicos, sabores y fragancias (Fdil et al., 1996; Crowell, 1997; Murphy et al., 2000; Allal et al., 2003; De Carvalho \& Da Fonseca, 2006a). En la oxidación del limoneno y el $\alpha$-pineno se presenta una competencia entre la oxidación del doble enlace y la oxidación en la posición alílica, debido a la presencia de los enlaces dobles de estas moléculas. El predominio de una reacción sobre la otra depende de la naturaleza de la olefina y de la relativa estabilidad de los radicales alílicos formados (Murphy et al., 2000).

La oxidación alílica se lleva a cabo por abstracción de un hidrógeno, preferiblemente en presencia de especies metálicas de bajo estado de oxidación (Murphy et al., 2000). Los procesos más utilizados para esta reacción son estequiométricos, basados en los sistemas $\mathrm{CrO}_{3}$ / piridina (Dauben et al., 1969) o $\mathrm{SeO}_{2}$ (Trachtenberg et al., 1970); sin embargo, se obtienen rendimientos bajos, se requieren reactivos costosos e indeseables desde el punto de vista ambiental y se generan subproductos organometálicos que deben ser recuperados o eliminados mediante procesos de purificación largos y costosos (Lempers \& Sheldon, 1996; Marwah \& Lardy, 1999). Utilizando $\mathrm{SeO}_{2}$ en cantidades catalíticas y agentes oxidantes como el peróxido de hidrógeno (Francis et al., 1976) y el hidroperóxido de terbutilo (TBHP) (Umbreit \& Sharpless, 1977), se evita la formación de compuestos organoselénicos, pero estos sistemas se deben mejorar (Singh et al., 1997). Mediante la reacción de Kharash-Sosnovsky se obtienen alcoholes alílicos por hidrólisis de un éster que se obtiene con perésteres como agentes oxidantes (Carloni et al., 2000; Eames \& Watkinson, 2001; Clark et al., 2004).

Los procesos catalíticos con cromo y complejos relacionados son los más eficientes para la oxidación alílica de alquenos (Sakthivel et al., 2003); sin embargo, su uso no es muy atractivo debido a la toxicidad de los catalizadores y los solventes requeridos. Con complejos de rutenio, rodio y paladio (Harre et al., 1998; Stultz et al., 2000; Moiseev \& Vargaftik, 2004; Catino et al., 2004) y con sales de bismuto (Salvador \& Silvestre, 2005) también se han obtenido altas actividades; no obstante, los costos de estos materiales no hacen viable su aplicación a escala industrial. El uso en catalizadores heterogéneos de especies activas menos costosas como hierro,

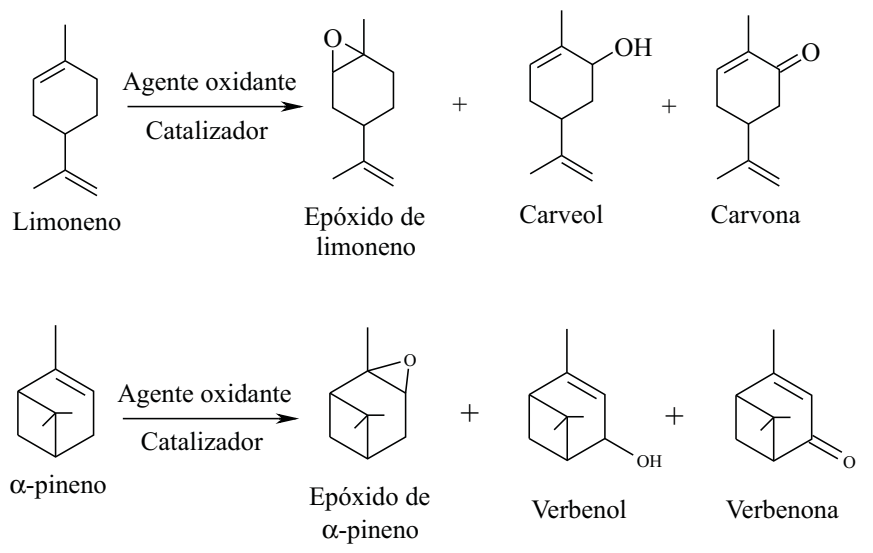

(a)

Figura 1. Productos de la oxifuncionalización de limoneno y $\alpha$-pineno. 
cobre o cobalto proporcionaría procesos de oxidación alílica más atractivos.

El carveol y la carvona se pueden obtener por oxidación alílica de limoneno, Figura 1a, y el verbenol y la verbenona por la oxidación alílica de $\alpha$-pineno, Figura $1 b$.

En nuestro grupo de investigación se evaluó la oxidación alílica de limoneno y $\alpha$-pineno con catalizadores biomiméticos a base de ftalocianinas metálicas inmovilizadas en sílica usando TBHP como oxidante. Se utilizaron varios grupos sustituyentes en la periferia de las ftalocianinas metálicas: $\mathrm{SO}_{3}^{-}, \mathrm{NO}_{2}^{-}, \mathrm{CH}_{2} \mathrm{Cl}^{-}, \mathrm{Cl}^{-}, \mathrm{y} \mathrm{F}^{-}$. Mediante la técnica UV-vis se identificaron especies monoméricas, diméricas y agregados en las ftalocianinas sintetizadas. Los estudios mecanísticos con los sistemas metaloftalocianina/ TBHP sugirieron que primero se forma el alcohol alílico, el cual luego se oxida a la cetona. El catalizador basado en el complejo de hierro, FePcCl ${ }_{16}-\mathrm{NH}_{2}-\mathrm{S}$, fue el más estable bajo las condiciones de reacción utilizadas en la oxidación alílica de limoneno y $\alpha$-pineno. Este material se pudo reutilizar al menos tres veces sin pérdida significativa de actividad (González, 2007).

En la oxidación alílica de limoneno con los sistemas metaloftalocianina / TBHP se obtuvieron conversiones de limoneno hasta del $88 \%$, pero alrededor del $13 \%$ de selectividad a carvona. La baja selectividad a carvona puede estar asociada con la gran cantidad de sitios que podrían ser atacados por especies de oxidación tipo radicales. Con complejos tetrasulfonados se encontró que la conversión de limoneno diminuyó con la electronegatividad del metal, en el siguiente orden: $\mathrm{Fe}>\mathrm{Co}>\mathrm{Cr}>\mathrm{Mn}$. La diferencia en actividad se puede atribuir al efecto del metal en la descomposición por radicales del TBHP y en la formación de las especies activas. La conversión de limoneno aumentó con el número de grupos extractores de electrones incorporados en la periferia de las ftalocianinas de hierro y manganeso, pero la distribución de los productos fue independiente del tipo de sustituyente. En el caso de la oxidación de $\alpha$-pineno con los sistemas metaloftalocianina / TBHP se observó un ataque preferencial de las especies oxidantes al carbono del doble enlace en comparación con el carbono en la posición alílica. Este comportamiento se atribuyó al solapamiento del hidrógeno alílico en la molécula. La presencia de grupos extractores de electrones no influyó significativamente en la actividad catalítica de la ftalocianina de hierro. La conversión de $\alpha$-pineno y el rendimiento del producto aumentaron apreciablemente con la adición de átomos de cloro en el catalizador de manganeso (González, 2007).

En esta revisión se presentan los principales procesos reportados para la obtención de carvona, carveol, verbenol y verbenona, haciéndose énfasis en los procesos catalíticos reportados en el período comprendido entre 1990 y 2007, especialmente aquellos que utilizan sistemas catalíticos biomiméticos. El carveol y la carvona se utilizan en industrias de sabores y de productos para higiene oral (Warshaw \& Zug, 1996; Quiroz et al., 1999; Gonçalves et al., 2006). El verbenol y la verbenona se usan en la industria alimenticia y como intermediarios en la síntesis del agente terapéutico taxol (Maksimchuk et al., 2005).

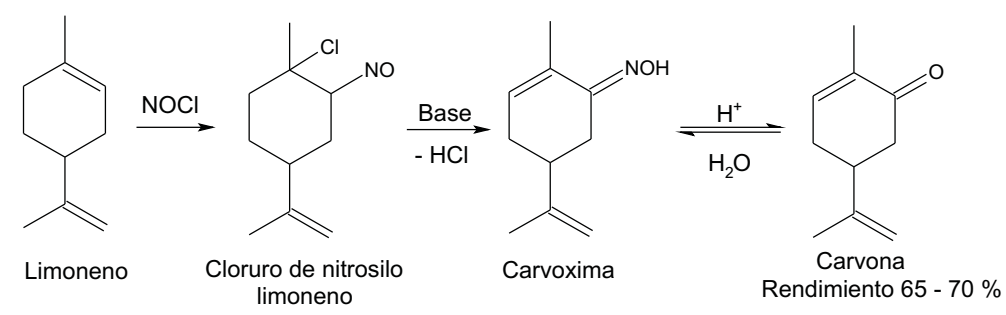

Figura 2. Síntesis de carvona a partir de limoneno mediante la ruta de la carvoxima. 


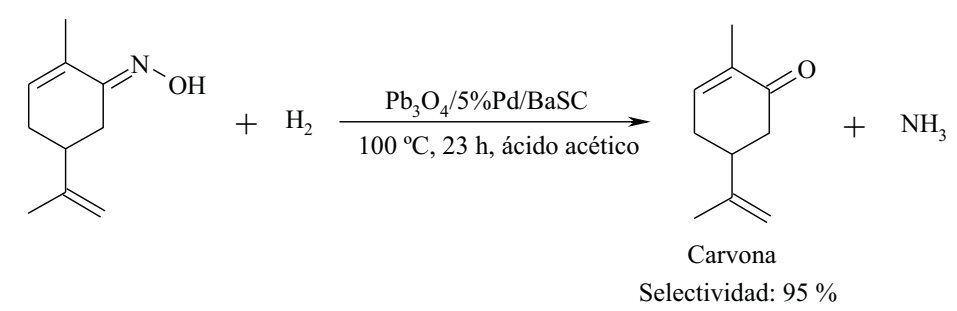

Figura 3. Hidrogenación de carvoxima sobre $5 \% \mathrm{Pd} / \mathrm{BaSC}$ impregnada con $\mathrm{Pb}_{3} \mathrm{O}_{4}$.

\section{Obtención de carvona, carveol, verbenol y verbenona}

\subsection{Obtención de carvona}

La carvona (p-menta-6,8-dien-2-ona) es una sustancia saborizante ampliamente utilizada en productos farmacéuticos, goma de mascar, pasta dental, enjuagues bucales, alimentos y bebidas (Rhodes \& Winskill, 1985; Davey et al., 2002; Carter et al., 2003; Pergentino et al., 2007). Adicionalmente, la carvona se conoce como agente fungicida, insecticida y antibacterial (Carter et al., 2003; De Carvalho \& Da Fonseca, 2006b; Tachibana et al., 2007). Los dos isómeros de la carvona, (S)-(+) y (R)-(-)-carvona, se encuentran en la naturaleza (Slotnick \& Bisulco, 2003). La (S)-(+)-carvona tiene aroma a alcaravea/eneldo y es el mayor constituyente del aceite de alcaravea y del aceite de semilla de eneldo. La (R)-(-)-carvona tiene aroma a hierbabuena y es el principal constituyente del aceite de hierbabuena (Slotnick \& Bisulco, 2003).

En la extracción (con hexano) de la carvona de semillas de alcaravea, se obtienen (Carter et al., 2003) rendimientos cercanos a $16.28 \mathrm{mg} /$ (g de semilla). El aceite de hierbabuena (derivado de Mentha spicata $L$ ) se produce principalmente en
Estados Unidos con algún desarrollo reciente en China y Suramérica. El mercado mundial de aceite de hierbabuena es aproximadamente de 1500 ton / año (Peterson \& Bienvenu, 2007); el precio de este aceite varía entre 20.41 y 34.46 US \$ / kg (MacTavish \& Harris, 2002), dependiendo de la presencia de residuos de pesticidas (Booth \& Klein, 1957; Peterson \& Bienvenu, 2007). También se ha reportado la presencia de carvona en extractos de Lippia alba (Mill.) en concentraciones entre 40.4 y $57.2 \%$ (Stashenko et al., 2004).

Debido a las limitaciones para la extracción de carvona (De Carvalho \& Da Fonseca, 2006b), se han estudiado varias rutas sintéticas para la producción de esta cetona a partir de limoneno, de su epóxido o de carveol. A continuación, se presentan los métodos más representativos que se han reportado para la síntesis de carvona.

\subsubsection{Obtención de carvona por la ruta de la carvoxima}

En la ruta de la carvoxima, Figura 2, el limoneno se trata con cloruro de nitrosilo para formar el cloruro de nitrosilo limoneno, que se deshidrohalogena a carvoxima; obteniéndose la carvona por hidrogenación de la carvoxima (Derfer et al., 1966).

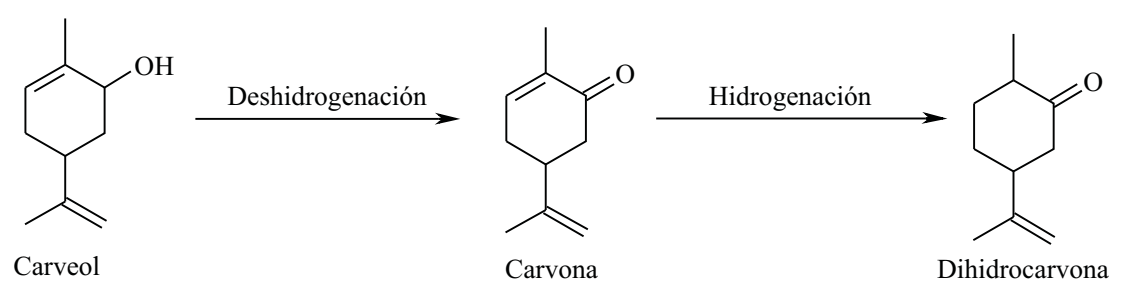

Figura 4. Obtención de dihidrocarvona por deshidrogenación de carveol. 


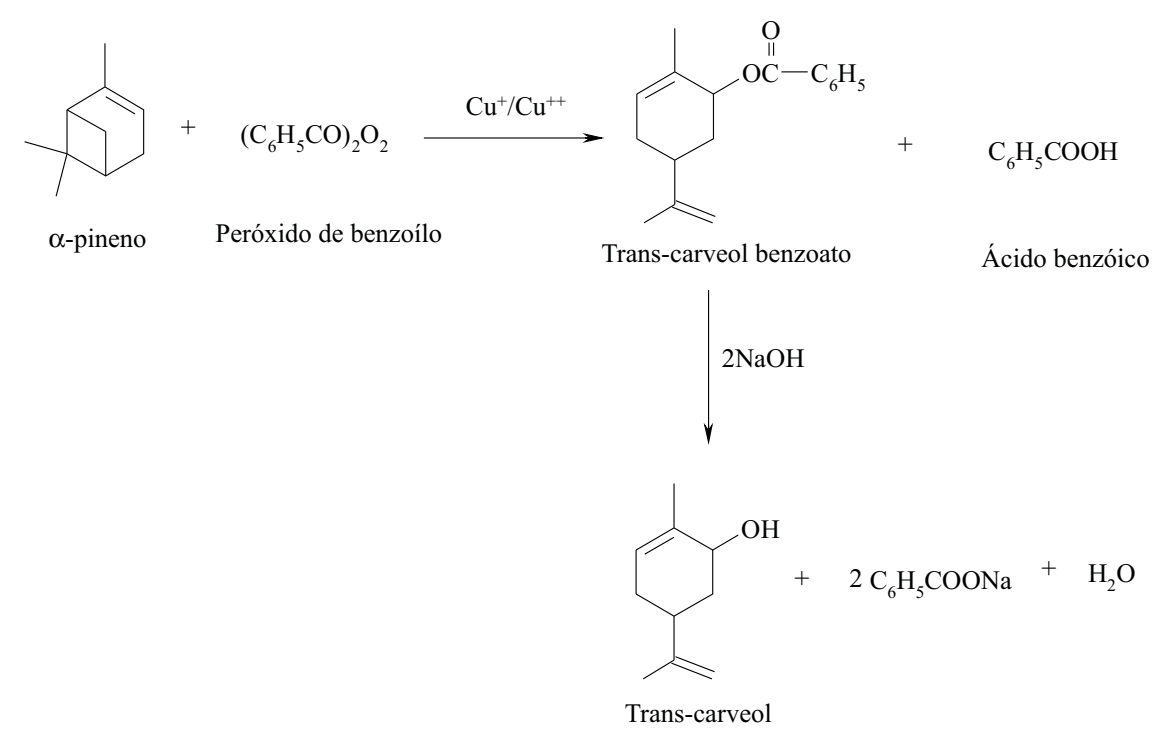

Figura 5. Síntesis de carveol a partir de a-pineno mediante la reacción de Kharash-Sosnovsky.

A pesar de los altos rendimientos a carvona se obtienen como subproductos el $\alpha$-terpineol, el cual es indeseable en aplicaciones de higiene oral por producir irritación de la mucosa nasal (Surferchicks, 2007) y cantidades estequiométricas de acetoxima, sustancia que se sospecha cancerígena. Adicionalmente, los efluentes contienen altas cantidades de sulfato, utilizado en la purificación de los productos. En el 2002 se patentó un proceso para la hidrogenación de carvoxima (Davey et al., 2002), Figura 3, con el catalizador $\mathrm{Pd} / \mathrm{BaSC}$ impregnado con $\mathrm{Pb}_{3} \mathrm{O}_{4}$ que evita la formación de productos indeseables como acetoxima o $\alpha$-terpineol. Con este método, se obtienen altos rendimientos a carvona, pero se requiere el uso de $\mathrm{NOCl}$ y de la mezcla de nitrato de sodio / $\mathrm{HCl}$ concentrado para la síntesis de carvoxima, lo cual presenta serios problemas ambientales y restricciones de seguridad (Ordonez et al., 2007).

\subsubsection{Obtención de carvona mediante deshidrogenación de carveol}

La carvona se puede obtener a partir de la deshidrogenación de carveol por la oxidación de Oppenauer o mediante un proceso catalítico en presencia de ácido sulfúrico. En la oxidación de Oppenauer se transfiere un hidrógeno desde el carveol a un compuesto carbonílico auxiliar por procesos catalíticos. Con el complejo 2,7-dimetil1,8-(bifenildioxi)bis(dialcoxialuminio) como catalizador y pivaldehído en cloruro de metilo como aceptor de hidrógeno, se reportan rendimientos a carvona del $91 \%$ (Ooi et al., 2002). Las principales desventajas de los procesos de oxidación de Oppenauer son la inestabilidad del catalizador en las condiciones de hidrólisis, el uso del compuesto carbonílico auxiliar en exceso y los largos e intensos procesos de separación $\mathrm{y}$ purificación de los productos.

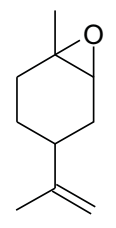

Epóxido

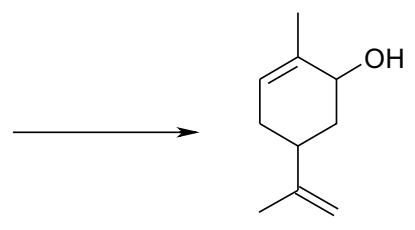

Alcohol alílico

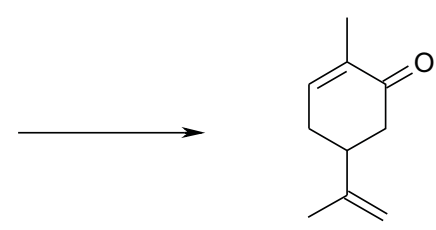

Carbonil $\alpha, \beta$-insaturado

Figura 6. Reagrupamiento del epóxido del limoneno a carveol y su posterior oxidación a carvona. 


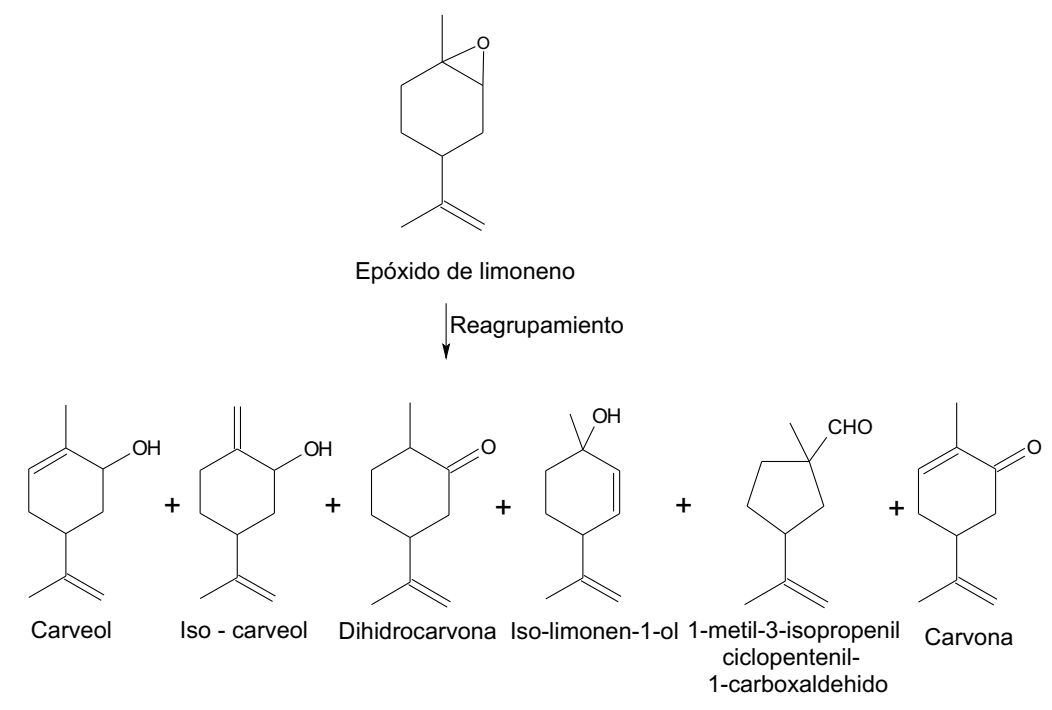

Figura 7. Productos posibles del reagrupamiento del epóxido de limoneno.

El segundo método de deshidrogenación de carveol (Kolomeyer \& Oyloe, 2005) requiere de catalizador y se realiza típicamente en ácido sulfúrico concentrado, obteniéndose rendimientos hasta del 93 \%. En general, los catalizadores utilizados son costosos o tóxicos y se requieren grandes excesos de agente oxidante. Adicionalmente, bajo las condiciones de reacción, el producto principal es la dihidrocarvona, ya que en medio ácido se produce una isomerización del doble enlace, y una posterior tautomería cetoenólica (Figura 4).

Se han utilizado varios complejos de metales de transición como catalizadores homogéneos de deshidrogenación. Con octoato de zinc $\left(230^{\circ} \mathrm{C}\right.$, $2 \mathrm{~h}$ ) se obtiene carvona con rendimientos del $79 \%$ y selectividad del $86 \%$. La selectividad a carvona aumenta hasta el $95 \%$ (10 h) con dodecano como solvente y eliminando el agua continuamente (Kolomeyer \& Oyloe, 2005). El uso de catalizadores homogéneos y las altas temperaturas son algunas de las principales desventajas de este método.

\subsection{Obtención de carveol}

\subsubsection{Reacción de Kharash-Sosnovsky}

En esta reacción se forma el carveol por hidrólisis del benzoato alílico que se obtiene con per-ésteres o peroxianhídridos como oxidantes estequiométricos (Eames \& Watkinson, 2001; Ooi et al., 2002; Clark et al., 2004). La reacción se lleva a cabo por mecanismos que involucran radicales y el centro metálico (cobre o cobalto) participa en la formación del enlace C-O (Eames y Watkinson, 2001). El $\alpha$-pineno se oxida con peróxido de benzó́lo en presencia de iones cuprosos o cúpricos; posteriormente, se hidroliza el éster benzoato a carveol (Figura 5).

\subsubsection{Isomerización de epóxidos}

El epóxido de $\alpha$-pineno puede reagruparse a carveol con un rendimiento del $45 \%$ con copolímeros de ácido 4-estirenosulfónico como catalizador (Motherwell et al., 2004). El epóxido de limoneno se reagrupa a carveol (Arata \& Tanabe, 1976; Gorzynski-Smith, 1984; Kolomeyer \& Oyloe, 2004), y éste se oxida a carvona (Kolomeyer \& Oyloe, 2005) como se muestra en la Figura 6. A continuación, se presentan los métodos que tradicionalmente se han utilizado para reagrupar los epóxidos a alcoholes alílicos.

- Apertura estequiométrica del anillo epoxídico con bases fuertes. Esta reacción se lleva a cabo con cantidades estequiométricas de reactivos costosos, como amida, diisopropilamida y butil-litio, amiduros de aluminio y butóxido de potasio. La Figura 7 presenta los posibles productos que pueden obtenerse de la apertura del epóxido de limoneno (Kolomeyer \& Oyloe, 2004). El iso-carveol es el producto principal 
cuando la abstracción del protón se lleva a cabo en el carbono menos sustituido, como cuando se utilizan bases fuertes (Gorzynski, 1984).

- Reagrupamiento catalítico del epóxido. Los alcóxidos de carveol se obtienen con selectividades del $24 \%$ y $60 \%$ en presencia de isopropóxido de aluminio e isobutóxido de titanio, respectivamente. Las desventajas comunes de estos procesos son los complicados procesos de purificación del producto final y la relativa baja actividad y selectividad.

El epóxido de limoneno se puede reagrupar con varios catalizadores. De los óxidos metálicos utilizados, el más activo para la producción de carveol (59\%) es $\mathrm{Al}_{2} \mathrm{O}_{3}$ (Arata \& Tanabe, 1976). Con alúmina-óxidos de tierras raras se obtiene carvona con una selectividad del $35 \%$ por la oxidación de Oppenauer de carveol, con dihidrocarvona y 1-metil-3-isopropenil ciclopentil-1-carboxaldehído como aceptores de hidrógeno. Con fosfato de litio, el epóxido de limoneno se reagrupa a cis-carveol con selectividad alrededor del $18 \%\left(57 \mathrm{~h}, 200{ }^{\circ} \mathrm{C}\right)$. El epóxido de limoneno se reagrupa a carveol con una selectividad del $84 \%$, con compuestos fenólicos como activadores o modificadores, salicilato de isopropilo y óxido de calcio $\left(200-300{ }^{\circ} \mathrm{C}, 6 \mathrm{~h}\right)$. La carvona se obtiene con un rendimiento del $63 \%\left(190-220^{\circ} \mathrm{C}, 9 \mathrm{~h}\right)$ por el reordenamiento del epóxido de limoneno con carbonato de zinc modificado con 2-nitrofenol en ciclohexanona (Kolomeyer \& Oyloe, 2004).

\subsubsection{Oxidación alílica de limoneno}

Los sistemas catalíticos más estudiados para la oxidación alílica de limoneno son los basados en sales de paladio y cobre; sin embargo, estos procesos se realizan en fase homogénea, involucran varias etapas de reacción o generan una gran cantidad de desechos tóxicos (Silva et al., 2002; Kala-Raj et al., 2003; Bussi et al., 2003; Gonçalves \& Gusevskaya, 2004). La oxidación alílica de limoneno también se ha llevado a cabo con complejos de metales de transición soportados, especialmente de cobalto, cromo, wolframio, vanadio y molibdeno.

- Catalizadores de paladio y cobre. El proceso Wacker que se realiza en presencia de sales de $\mathrm{PdCl}_{2}-\mathrm{CuCl}_{2}$ en medio acuoso se utiliza comúnmente a escala industrial para la oxidación de etileno a acetaldehído (Popp et al., 2006).

El proceso incluye la oxidación estequiométrica del alqueno con $\mathrm{Pd}^{\mathrm{II}}$ y la posterior reoxidación del $\mathrm{Pd}^{0}$ con oxígeno, en presencia de sales de cobre, Ecs. (1)-(3) (Sheldon \& Kochi, 1981, p. 190-191; Firdoussi et al., 1992; Gusevskaya \& Gonçalves, 1997; Bussi et al., 2003).

$$
\begin{aligned}
& \mathrm{C}_{2} \mathrm{H}_{4}+\mathrm{PdCl}_{2}+\mathrm{H}_{2} \mathrm{O} \rightarrow \mathrm{CH}_{3} \mathrm{CHO}+\mathrm{Pd}+2 \mathrm{HCl} \\
& \mathrm{Pd}+2 \mathrm{CuCl}_{2} \rightarrow \mathrm{PdCl}_{2}+\mathrm{Cu}_{2} \mathrm{Cl}_{2} \\
& \mathrm{Cu}_{2} \mathrm{Cl}_{2}+2 \mathrm{HCl}+1 / 2 \mathrm{O}_{2} \rightarrow 2 \mathrm{CuCl}_{2}+\mathrm{H}_{2} \mathrm{O}
\end{aligned}
$$

\begin{tabular}{|c|c|c|c|c|c|c|c|}
\hline Entrada & Catalizador & Oxidante & Solvente & $\begin{array}{l}\mathrm{T},{ }^{\circ} \mathrm{C} \\
(\mathrm{t}, \mathrm{h})\end{array}$ & $\%$ conv. & $\begin{array}{l}\text { Carvona } \\
\% \mathrm{~S}\end{array}$ & $\begin{array}{l}\text { Carveol } \\
\% \mathrm{~S}\end{array}$ \\
\hline \multirow[t]{2}{*}{1} & $\mathrm{Li}_{2} \mathrm{PdCl}_{4}-$ & - & $\mathrm{H}_{2} \mathrm{O} /$ Acetona $(1: 1)$ & $80(24)$ & 79 & 4 & 2 \\
\hline & $\mathrm{CuCl}_{2}$ & & & & & & \\
\hline \multirow[t]{2}{*}{2} & $\mathrm{Li}_{2} \mathrm{PdCl}_{4}$ & Benzoquinona/ & $\mathrm{H}_{2} \mathrm{O} /$ Acetona $(1: 1)$ & $80(7)$ & 90 & 73 & 5 \\
\hline & & PTSA & & & & & \\
\hline 3 & $\mathrm{PdCl}_{2}-\mathrm{CuCl}_{2}$ & $\mathrm{O}_{2}$ & Acido acético & $50(24)$ & 17 & 6 & 33 \\
\hline 4 & $\mathrm{PdCl}_{2}-\mathrm{CuCl}_{2}$ & TBHP & Terbutanol & $30(24)$ & 67 & 13 & 6 \\
\hline
\end{tabular}

Tabla 1. Sistemas homogéneos basados en Pd para la oxidación alílica de limoneno.

$\%$, porcentaje selectividad. \% conv., porcentaje de conversión. PTSA: ácido para-toluensulfónico. 
Algunas modificaciones del proceso Wacker para la oxidación alílica de limoneno se presentan en la Tabla 1 (Firdoussi et al., 1998; Silva et al., 2002; Kala-Raj et al., 2003). El cloruro de litio aumenta la solubilidad del paladio por la formación de $\mathrm{Li}_{2} \mathrm{PdCl}_{4}$ (Firdoussi et al., 1998), aumentando la conversión a $80 \%$ pero con selectividad baja hacia la carvona y el carveol (entrada 1 en la tabla). La utilización de benzoquinona como donador de oxígeno y ácido para-toluensulfónico (PTSA) como co-oxidante (entrada 2), mejoró significativamente la selectividad hacia la carvona; sin embargo, por razones ambientales no es recomendable el uso de benzoquinona y PTSA. Con $\mathrm{PdCl}_{2}-\mathrm{CuCl}_{2}$, oxígeno molecular y TBHP, se obtuvieron acetatos y derivados terbutilperoxi (Silva et al., 2002) como subproductos (entradas 3 y 4 , respectivamente).

El Pd inmovilizado en matrices inorgánicas como $\mathrm{SiO}_{2}$ sol-gel (Gonçalves \& Gusevskaya, 2004; Gonçalves et al., 2005; Robles-Dutenhefner et al., 2004a) e hidrotalcitas (Bussi et al., 2003) ha sido utilizado para la oxidación alílica de limoneno.

Tabla 2. Sistemas catalíticos basados en Co y otros metales para la oxidación alílica de limoneno.

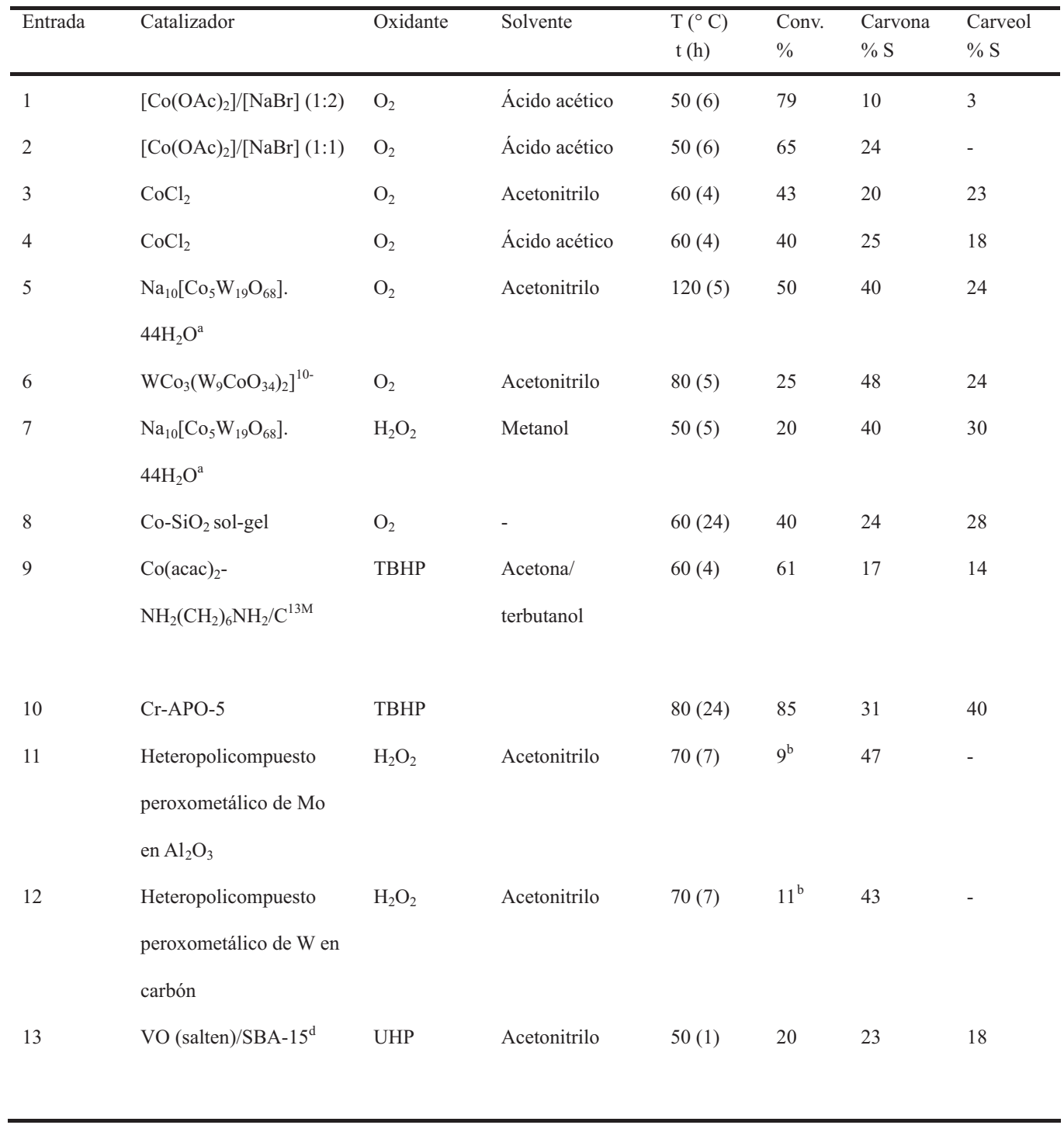

a Sales sódicas de cobalto con polioxotungstatos tipo sándwich.

b Máxima conversión posible: $34.6 \%$. 
Con $\mathrm{Pd}-\mathrm{SiO}_{2}$ en presencia de $\mathrm{CuCl}_{2}$ y $\mathrm{LiCl}$, ácido acético y $\mathrm{O}_{2}$ (Robles-Dutenhefner et al., 2004a), se obtuvo una conversión de limoneno del 90 \% y acetatos alílicos con selectividades cercanas al $75 \%$. Con $\mathrm{Cu}$ y $\mathrm{Pd}$ soportados en hidrotalcitas y oxígeno molecular como agente oxidante (Bussi et al., 2003), la conversión del limoneno fue de aproximadamente $60 \%$ con una relación epóxido / (carveol + carvona) de 2.1. Este sistema aparentemente no sigue un mecanismo tipo Wacker, ya que el cobre participa en la descomposición de los hidroperóxidos formados y el paladio activa los enlaces químicos (Bussi et al., 2003).

- Catalizadores de cobalto. El cobalto es uno de los catalizadores más utilizados en los procesos de oxidación con oxígeno molecular, proceso denominado de autoxidación que se lleva a cabo por radicales libres (Carey \& Sundberg, 1990, p. 222-225), como se muestra en la Tabla 2 (entradas 1-6, 8). Con oxígeno molecular como oxidante, las mayores conversiones se obtienen en presencia de bromuro de sodio, entradas 1 y 2 , debido a la formación de especies $\mathrm{BrCo}{ }^{\text {III }} \mathrm{OAc}$ que incrementan la abstracción de hidrógenos en la molécula; sin embargo, la selectividad disminuye debido a la bromación del limoneno (Gomes \& Antunes, 1997).

La actividad del catalizador $\mathrm{CoCl}_{2}$ es similar con acetonitrilo y ácido acético (entradas 3 y 4 de la Tabla 2) (Da Silva et al., 2003). La selectividad más alta a los productos de oxidación alílica con catalizadores de Co se obtuvo (entradas 5 -7) con los polyoxotungstatos tipo sándwich (Kala-Raj et al., 2003).

Con el catalizador heterogéneo $\mathrm{Co}-\mathrm{SiO}_{2}$ sol-gel se requirió un tiempo mayor de reacción (entrada 8), comparado con el catalizador homogéneo (entrada 3). Del epóxido de limoneno se obtuvieron derivados poliméricos (entrada 9) (selectividad $>30 \%$ ) con el catalizador $\mathrm{Co}(\mathrm{acac})_{2}-\mathrm{NH}_{2}\left(\mathrm{CH}_{2}\right)_{6} \mathrm{NH}_{2} / \mathrm{C}^{13 \mathrm{M}}$ (Oliveira et al., 2005).

- Catalizadores de cromo, wolframio, molibdeno o vanadio. Aunque con el catalizador heterogéneo Cr-APO-5 (Tabla 2, entrada 10)

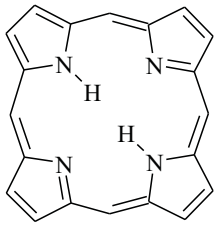

(a)

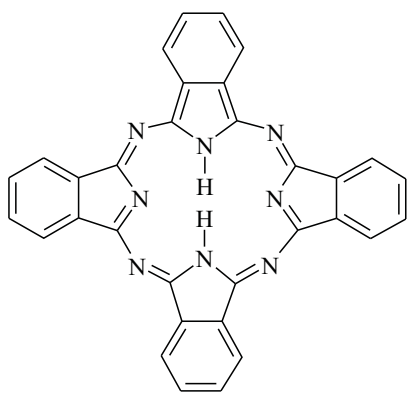

(b)

Figura 8. Estructuras básicas de porfirinas (a) y ftalocianinas (b).

(Lempers \& Sheldon, 1996) se obtuvo una conversión alta de limoneno, el catalizador presentó lixiviación del cromo. Con catalizadores de molibdeno y wolframio soportados en alúmina o carbón (Casuscelli et al., 2004), se obtuvo (entradas 11 y 12) una conversión de limoneno del $11 \%$ (para una conversión máxima posible de $34.6 \%$ ). El epóxido se isomerizó a carvona dada la acidez del catalizador y la presencia de agua en el medio de reacción. Con el complejo de vanadio soportado en el material mesoporoso SBA-15 y un agente oxidante anhidro, UHP, (Trissa \& Halligudi, 2005), la selectividad a los productos de oxidación alílica fue del $41 \%$ (entrada 13).

\subsubsection{Sistemas biocatalíticos}

La oxidación alílica de limoneno también se ha llevado a cabo con biocatalizadores (Noma et al., 1992; Savithiry \& Oriel, 1998; Mikami, 1988); sin embargo, su uso es restringido debido a la biotoxicidad del limoneno. Con la bacteria termófila Bacillus Stearothermophilus BR388 clonada en Escherichia coli (Savithiry \& Oriel, 1998) se obtuvieron el $\alpha$-terpineol y la carvona bajo condiciones bifásicas $\left(50{ }^{\circ} \mathrm{C}, 72 \mathrm{~h}\right)$ a concentraciones de $235 \mathrm{mg} / \mathrm{L}$ y $35 \mathrm{mg} / \mathrm{L}$, respectivamente. Con la enzima $\mathrm{P}-450$ limoneno6-hidroxilasa en membranas de Escherichia coli se obtuvo trans-carveol con un rendimiento del $99 \%$ (Haudenschild et al., 2000). Duetz et al. (2000) reportaron la biotransformación de $d$-limoneno a $(+)$ trans-carveol (rendimiento $94 \%$ ) y a (+) carvona en trazas, con Rhodococcus opacus cultivada en un medio mineral con glucosa y tolueno. 


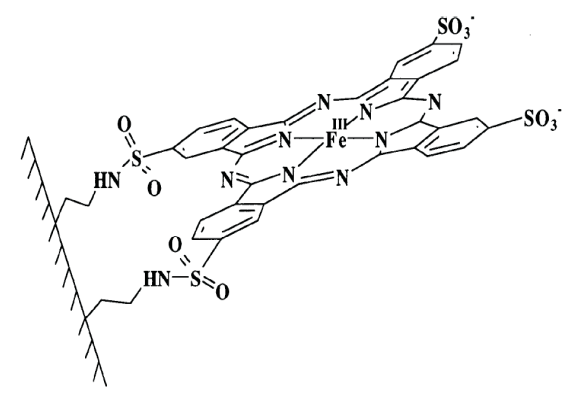

(a)

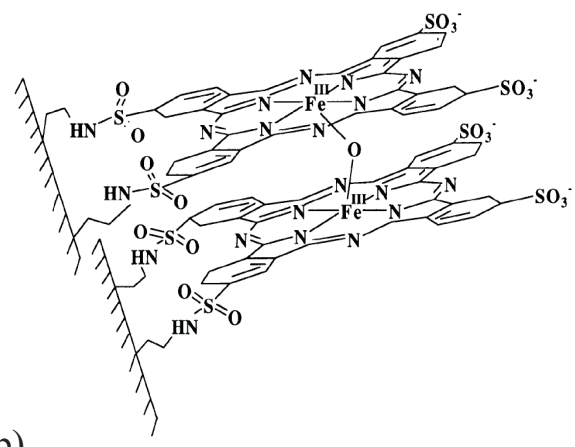

(b)

Figura 9. Catalizadores FePcS monomérico (a) y dimérico (b) soportados en sílica.

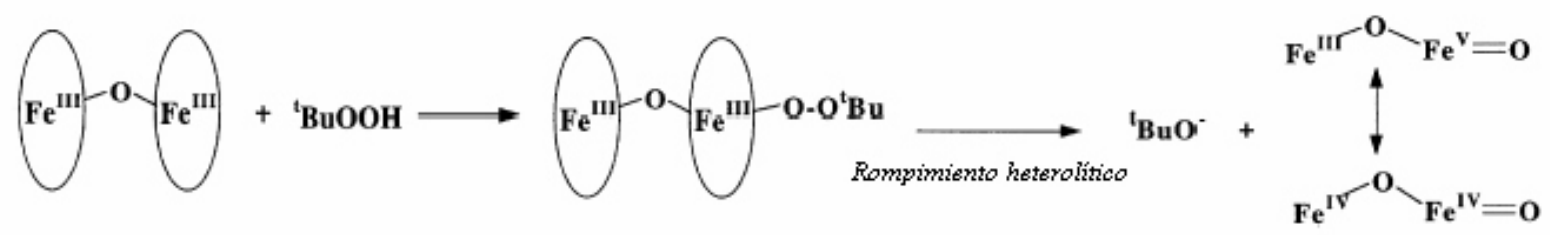

Figura 10. Esquema propuesto para la formación de especies activas entre d-FePcSy TBHP

Tabla 3. Actividad catalítica de $\mathrm{FePcS}$ y $\mathrm{FePcS}-\mathrm{SiO}_{2}$ con TBHP en la oxidación alílica de limoneno ${ }^{a}$

\begin{tabular}{|c|c|c|c|c|c|c|c|}
\hline \multirow{2}{*}{ Entrada } & \multirow{2}{*}{ Catalizador } & \multirow{2}{*}{ Solvente } & \multirow{2}{*}{ Temperatura, ${ }^{\circ} \mathrm{C}$} & \multirow{2}{*}{ Conversión, \% } & \multicolumn{3}{|c|}{ Rendimiento $\%$} \\
\hline & & & & & LE & $\mathrm{C}$ & $\mathrm{CA}$ \\
\hline 1 & FePcS & $\mathrm{CH}_{3} \mathrm{CN}$ & 40 & 55 & 4 & 3 & 4 \\
\hline 2 & FePcS-S & $\mathrm{CH}_{3} \mathrm{CN}$ & 40 & 80 & 3 & 3 & 12 \\
\hline 3 & FePcS-S & Acetona & 40 & 55 & 4 & 8 & 15 \\
\hline 4 & FePcS-S & Acetato de etilo & 40 & 65 & 6 & 3 & 9 \\
\hline 5 & FePcS-S & $\mathrm{CH}_{3} \mathrm{CN}$ & 60 & 77 & 3 & 5 & 15 \\
\hline
\end{tabular}

S: $\mathrm{SiO}_{2}$, LE: epóxido de limoneno, C: carveol, CA: carvona. ${ }^{\mathrm{a}}$ Condiciones de reacción: $4 \mathrm{~mL}$ de substrato en solvente $0.125 \mathrm{M}, 0.5 \%$ mol catalizador, $1.3 \mathrm{mmol}$ en $\mathrm{CH}_{2} \mathrm{Cl}_{2}(3.5 \mathrm{M}), 24 \mathrm{~h}$. ${ }^{\mathrm{b}}$ Rendimiento $=($ mol de producto $(\mathrm{LE}, \mathrm{C}, \mathrm{CA})) /($ moles iniciales de limoneno $)$.

\subsubsection{Sistemas catalíticos biomiméticos}

Una de las reacciones más importantes en el metabolismo de los seres vivos es la oxidación, la cual es catalizada por enzimas (Salavati-Niassary et al., 2000). Con el fin de mimetizar los sitios activos enzimáticos, especialmente de las enzimas monooxigenasas de la familia citocromo P-450, se han investigado las porfirinas sintéticas (Figura 8a) y las ftalocianinas (Figura 8b) (Wang et al., 1999; Salavati-Niassary et al., 2000).

Los catalizadores de oxidación más promisorios son los complejos de hierro con diferentes ligandos, usualmente $N$-bases cíclicas quelatadas (Georgiy et al., 2004). Por el precio y la poca toxicidad del hierro, los complejos de este metal se han utilizado como catalizadores en reacciones de epoxidación y oxidación alílica (González et al., 2004; Maksimchuk et al., 2005).

Como modelos de la acción catalítica de la enzima citocromo P-450 en reacciones de oxidación, se han estudiado los complejos sintéticos de porfirinas de hierro. Como las porfirinas se degradan bajo las condiciones de reacción, se prefieren los complejos de metaloftalocianinas $(\mathrm{MePc})$ que tienen una estructura similar a la de las 
porfirinas y son más económicos y estables a la degradación (Grootboom \& Nyokong, 2002). En general, las $\mathrm{MePc}$ de $\mathrm{Fe}$, Mn y Co son activas para la oxidación de olefinas con $\mathrm{H}_{2} \mathrm{O}_{2}$ y TBHP como agentes oxidantes (Weber et al., 1993; Safari \& Bahadoran, 2001; Sorokin et al., 2002).

Es posible obtener catalizadores heterogéneos activos mediante la inmovilización covalente de $\mathrm{MePc}$ en la superficie de sílicas, ya que de esta forma es posible controlar la síntesis de los catalizadores y obtener diferentes especies activas (Ernst et al., 1997; Sorokin \& Tuel, 1999; Ganschow et al., 1999; Sorokin et al., 2002; Karandikar et al., 2006).

La tetrasulfoftalocianina de hierro (III), FePcS, se ha inmovilizado en sílica (Sorokin \& Tuel, 1999; Sorokin \& Tuel, 2000) en su forma monomérica $\left(\mathrm{m}-\mathrm{FePcS}-\mathrm{SiO}_{2}\right)$ y dimérica (d-FePcS-SiO $\left.{ }_{2}\right)$ como se muestra en las Figuras $9 \mathrm{a}$ y 9b, respectivamente. Estos catalizadores se utilizaron en la oxidación de 2,3,6-trimetilfenol con TBHP, a 1,4-trimetilbenzoquinona (rendimientos superiores al $80 \%$ ), precursora de la vitamina $\mathrm{E}$ (Sorokin et al., 2002).

La forma dimérica de la ftalocianina de hierro $\mathrm{FePcS}$, d-FePcS, sin inmovilizar e inmovilizada en sílica, se ha utilizado en la oxidación alílica de limoneno (Tabla 3) con TBHP (Pérollier \& Sorokin, 2002; González et al., 2004; González et al., 2005; González et al., 2006). El ataque preferencial del enlace activo $\mathrm{C}-\mathrm{H}$ sobre el enlace $\mathrm{C}=\mathrm{C}$ con el sistema catalítico d-FePcS/TBHP sugiere que se lleva a cabo una ruptura heterolítica del TBHP con una posterior formación de especies $\mathrm{de} \mathrm{Fe}^{\mathrm{IV}}$ (Sorokin \& Tuel, 2000) como se muestra en la Figura 10. Estas especies son las que promueven la abstracción de hidrógenos alílicos (Weber et al., 1993).

Al inmovilizar el complejo FePcS en sílice, se observa un aumento en la conversión del sustrato y en el rendimiento de la cetona (Tabla 3, entradas 1 y 2), lo cual indica que las especies diméricas son activas para esta reacción (Zefírov \& Zakharov, 1998). La conversión más alta de limoneno con FePcS-S a $40{ }^{\circ} \mathrm{C}$ se obtiene con acetonitrilo (entrada 2) y ésta no aumenta al incrementar la temperatura (entrada 5). Con acetona (entrada 3) se obtiene la mayor selectividad a los productos de oxidación alílica, especialmente a carvona (27\%).

\subsection{Obtención de verbenol y verbenona}

El verbenol y la verbenona se pueden obtener mediante la oxidación alílica de $\alpha$-pineno (ver Figura 1). El verbenol se utiliza en la preparación de fragancias (Simakova \& Semikolenov, 2003) e insecticidas (Holsten et al., 2000). La verbenona tiene un olor característico que recuerda al canfor, mentol y apio. Esta cetona es útil en la preparación de saborizantes, insecticidas y drogas (Dulou et al., 1977; Ohloff \& Giersch, 1979; Wender \& Mucciaro, 1992; Murphy et al., 2000; Corvi-Mora \& Ranise, 2003; Marwah \& Lardy, 2004; Passaro $\&$ Webster, 2004). La verbenona también se utiliza como material de partida para la síntesis de taxol, agente importante en el tratamiento del cáncer de ovarios y el cáncer de mama (Wender \& Mucciaro, 1992; Murphy et al., 2000; Trissa et al., 2002). La verbenona es el principal componente de Spanish verbena, de ahí el nombre de los aceites Spanish Eucalyptus globulus y Spanish Rosmarinus officinalis (28.9\% verbenona) (Ravid et al., 1997). La verbenona extraída es costosa e insuficiente para su demanda, además el proceso para su extracción es tedioso. Se han reportado varios sistemas para la oxidación alílica de $\alpha$-pineno, pero debido al mecanismo de radicales de esta reacción no se ha encontrado un sistema selectivo al verbenol y verbenona.

Con el proceso no catalítico patentado (Marwah \& Lardy, 2000; Marwah \& Lardy, 2004) que utiliza peryodato de sodio y TBHP se obtiene verbenona con un rendimiento del $16 \%$; aunque la reacción se lleva a cabo a temperatura ambiente, tiene la desventaja de requerir piridina. En otro sistema reportado por los mismos autores (Marwah \& Lardy, 2002) se utilizan compuestos de cromo y como cooxidante una imida ácida $N$-hidroxi dicarboxilica, obteniéndose verbenona con un rendimiento hasta del $50 \%$. Adicionalmente, la oxidación alílica de $\alpha$-pineno puede llevarse a cabo con microorganismos o con sistemas catalíticos homogéneos y heterogéneos a base de cobalto, cromo, rutenio, cobre, paladio y titanio y TBHP como agente oxidante. 
Tabla 4. Oxidación alílica de $\alpha$-pineno con catalizadores de cobalto al $0.15 \%$.

\begin{tabular}{llllll}
\hline Entrada & Catalizador & Oxidante & $\mathrm{T},{ }^{\circ} \mathrm{C}$ & $\mathrm{t}, \mathrm{h}$ & Rendimiento a \\
& & & & & verbenona, $\%$ \\
\hline 1 & {$\left[\mathrm{Co}(\text { piridina })_{2} \mathrm{Br}_{2}\right]$} & $\mathrm{O}_{2}$ & 100 & 23 & 32 \\
2 & {$\left[\mathrm{Co}(4-\text {-metilpiridina })_{2} \mathrm{Br}_{2}\right]$} & $\mathrm{O}_{2}$ & 100 & 23 & 32 \\
3 & {$\left[\mathrm{Co}(4-\text { metilpiridina })_{2} \mathrm{Br}_{2}\right]$} & $\mathrm{O}_{2} /(\mathrm{TPBP})^{\mathrm{a}}$ & 100 & 23 & 40 \\
4 & {$\left[\mathrm{Co}(4-\text { metilpiridina })_{2} \mathrm{Br}_{2}\right]$} & $\mathrm{O}_{2} /(\mathrm{TBHP})^{\mathrm{b}}$ & 60 & 23 & 65 \\
5 & $\mathrm{Co}\left(\mathrm{NO}_{3}\right)_{2}$ & $\mathrm{O}_{2}$ & 100 & 23 & 45 \\
6 & $\mathrm{Co}\left(\mathrm{NO}_{3}\right)_{2}$ & $\mathrm{O}_{2} /(\mathrm{TBHP})^{\mathrm{a}}$ & 60 & 23 & 35 \\
7 & $\mathrm{CoBr} /$ ácido acético & $\mathrm{O}_{2} /(\mathrm{TBHP})^{\mathrm{a}}$ & 60 & 24 & 39 \\
8 & $\mathrm{CoCl}_{2} /$ ácido acético & $\mathrm{O}_{2} /(\mathrm{TBHP})^{\mathrm{a}}$ & 60 & 23 & 24 \\
\hline
\end{tabular}

${ }^{\mathrm{a}} 11.5 \% \mathrm{~mol} .{ }^{\mathrm{b}} 115 \% \mathrm{~mol}$
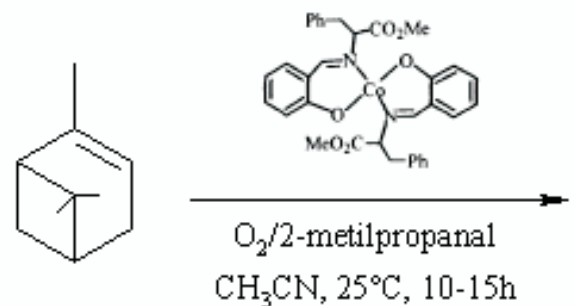

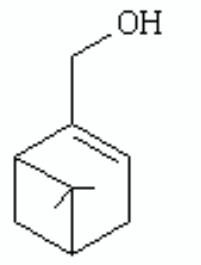

Mirtenol<smiles>C=C1C(O)CC2CC1C2(C)C</smiles>

Pinocarveol

Rdto: $50 \%$

Figura 11. Oxidación alílica de $\alpha$-pineno con complejos de cobalto Schiff.

\subsubsection{Catalizadores de Co}

Se reportaron varios complejos de cobaltopiridina para la autoxidación de $\alpha$-pineno en ausencia de solvente (Lajunen \& Koskinen, 1994). Con el complejo $\mathrm{Co}\left(4-\mathrm{Me}\left(\mathrm{C}_{5} \mathrm{H}_{4} \mathrm{~N}\right)_{2} \mathrm{Br}_{2}\right)$, el rendimiento a verbenona fue del $72 \%$ ( 7 días, $\left.72{ }^{\circ} \mathrm{C}\right)$; el producto se dimerizó al separarlo por destilación con vapor en una solución con bisulfito a $\mathrm{pH} \sim 7$, y el rendimiento disminuyó a $35 \%$. En la oxidación alílica de $\alpha$-pineno con complejos de cobalto, Tabla 4, se observa que la combinación de $\mathrm{O}_{2}$ con TBHP tiene un efecto positivo en la formación de verbenona (entradas 3 y 4); el TBHP actúa como iniciador de la abstracción de hidrógeno y su efecto aumenta al utilizarse en exceso (entrada 4). La verbenona en este caso, se obtiene por la oxidación del alcohol alílico formado inicialmente (Lajunen, 2001). Uno de los mayores inconvenientes de los sistemas que utilizan complejos cobalto-piridina es la utilización de cantidades estequiométricas de estos complejos y la formación de residuos tóxicos (Trissa et al., 2002).

Entre los catalizadores $\mathrm{CoCl}_{2}, \mathrm{CoBr}_{2}$ y $\mathrm{Co}\left(\mathrm{NO}_{3}\right)_{2}$ (Tabla 4, entradas 5-8) (Lajunen et al., 2003), la sal $\mathrm{Co}\left(\mathrm{NO}_{3}\right)_{2}$ fue más activa que los complejos cobalto-piridina a $100{ }^{\circ} \mathrm{C}$ con $\mathrm{O}_{2}$ (entrada 5); los mayores rendimientos a verbenona se obtuvieron con $\mathrm{CoBr}_{2}$ / ácido acético (entrada 7). El mecanismo propuesto para la oxidación de $\alpha$-pineno con TBHP y catalizadores de Co, sugiere que el TBHP promueve la transformación del 
Tabla 5. Oxidación alílica de $\alpha$-pineno con catalizadores de rutenio y titanio.

\begin{tabular}{llllll}
\hline Entrada & Catalizador & Oxidante & Solvente & $\mathrm{T},{ }^{\circ} \mathrm{C}$ & Rendimiento a \\
& & & $(\mathrm{t}, \mathrm{h})$ & verbenona, $\%$ \\
\hline 1 & $\mathrm{Ru}(\mathrm{TAZS})(\mathrm{p}$-cimeno) & $\mathrm{O}_{2} /$ isobutiraldehído & $\mathrm{CH}_{2} \mathrm{Cl}_{2}$ & $\mathrm{~T} . \mathrm{A}(4)$ & 16 \\
3 & $\mathrm{Ru}(\mathrm{III})$ saloph-Y & $\mathrm{O}_{2} / \mathrm{AIBN}$ & $\mathrm{CH}_{3} \mathrm{CN}$ & $100(3)$ & 11 \\
4 & $\mathrm{Ti}-\mathrm{MCF}$ & $\mathrm{H}_{2} \mathrm{O}_{2}$ & $\mathrm{CH}_{3} \mathrm{CN}$ & $30(5)$ & 10 \\
\hline
\end{tabular}

Ru(TAZS): Ru-1,2,4-triazepinas, AIBN: azobis-isobutironitrilo, T.A: temperatura ambiente

sustrato incluso en ausencia de catalizador (Lajunen et al., 2003). Con Co(acac) $)_{2}$ y TBHP, en ausencia de $\mathrm{O}_{2}$ (Allal et al., 2003), los principales productos de reacción son hidroperóxidos (rendimientos del $70 \%$ ); sin embargo, al burbujear oxígeno y adicionar el TBHP lentamente se obtiene verbenona con un rendimiento del $78 \%$ (acetonitrilo, $70{ }^{\circ} \mathrm{C}$ ). En la autoxidación de $\alpha$-pineno con el catalizador heterogéneo $\mathrm{Co}-\mathrm{SiO}_{2}$ sol-gel se obtuvo una conversión de $40 \%$ con selectividades a verbenona y verbenol de $34 \%$ y $26 \%$, respectivamente (Robles-Dutenhefner et al., 2004b).

Con 2-metilpropanal como aldehído de sacrificio en el sistema catalítico $\mathrm{Co}^{(\mathrm{II})}$ en complejos de bases de Schiff $/ \mathrm{O}_{2}$, se favorece la formación del epóxido cuando el complejo es de naturaleza iónica y la formación de mirtenol y pinocarveol (Figura 11) cuando el complejo es neutro (Reddy et al., 1995).

\subsubsection{Catalizadores de $\mathrm{Cr}$}

El cromo ha sido uno de los metales más utilizados en reacciones de oxidación, pero su uso es restringido dado su carácter tóxico. Con Cr-APO $4^{-5}$ (Lempers \& Sheldon, 1996) y Cr soportado en montmorillonita (Choudary et al., 1992) con TBHP se obtuvieron rendimientos a verbenona superiores al $60 \%$; sin embargo, en ambos casos se presentó lixiviación del cromo (Sheldon et al., 1997; Sheldon et al., 1998). En titania-sílice impregnada con $\mathrm{Cr}$ se encontró un efecto sinergístico entre el titanio y el cromo (VI) (McMorn et al., 2000), obteniéndose verbenona con una selectividad del $60 \%$, pero no se reportaron datos de estabilidad del $\mathrm{Cr}$ en el catalizador.

\subsubsection{Catalizadores basados en Ru y Ti}

En la Tabla 5 se presenta la actividad de otros sistemas catalíticos en la oxidación alílica de $\alpha$-pineno. Con complejos basados en $\mathrm{Ru}$-1,2,4-triazepinas e isobutiraldehído- $\mathrm{O}_{2}$ con el sistema de Mukaiyama (entrada 1), se obtuvo (Fdil et al., 2002) conversión completa del sustrato y el principal producto fue el epóxido (selectividad de $82 \%$ ), la selectividad a la verbenona fue del $16 \%$. Con complejos saloph (o-fenilenbis (salicilideniminato)) de rutenio, $\mathrm{O}_{2}$ y azobisisobutironitrilo (AIBN) como iniciador de radicales (entrada 2) (Trissa et al., 2002), el rendimiento a verbenona fue menor (11\%). Con $\mathrm{H}_{2} \mathrm{O}_{2}$ como agente oxidante y los catalizadores TiMCF (del inglés Mesoporous Cellular Foam) y $\mathrm{H}_{5} \mathrm{PW}_{11} \mathrm{TiO}_{40} / \mathrm{SiO}_{2}$ (entradas 3 y 4 ), los rendimientos a verbenona fueron del $10 \%$. La actividad del heteropoliácido se mantuvo en por lo menos ocho reutilizaciones (Maksimchuk et al., 2005).

\subsubsection{Sistemas biocatalíticos}

La verbenona se ha obtenido directamente del a-pineno con hongos como el Aspergillus niger (glucosa 0.6 M, pH de 7) por hidroxilación del $\alpha$-pineno a verbenol y posterior deshidrogenación de este a verbenona, con un rendimiento a la cetona del $17 \%$ (Agrawal \& Joseph, 2000). En la biotransformación de $\alpha$-pineno en un medio celular de Picea abies el verbenol fue el producto 
Tabla 6. Actividad catalitica de PcS y FePcS-S con TBHP en la oxidación alílica de $\alpha$-pineno ${ }^{a}$.

\begin{tabular}{|c|c|c|c|c|c|c|c|}
\hline \multirow{2}{*}{ Entrada } & \multirow{2}{*}{ Catalizador } & \multirow{2}{*}{ Solvente } & \multirow{2}{*}{ Temperatura, ${ }^{\circ} \mathrm{C}$} & \multirow{2}{*}{ Conversión, $\%$} & \multicolumn{3}{|c|}{ Rendimiento ${ }^{\mathrm{b}} \%$} \\
\hline & & & & & $\mathrm{PE}$ & $\mathrm{V}$ & $\mathrm{VE}$ \\
\hline 1 & $\mathrm{FePcS}$ & $\mathrm{CH}_{3} \mathrm{CN}$ & 40 & 94 & 5 & 8 & 20 \\
\hline 2 & FePcS-S & $\mathrm{CH}_{3} \mathrm{CN}$ & 40 & 94 & 1 & 6 & 17 \\
\hline 3 & FePcS-S & Acetona & 40 & 75 & 1 & 6 & 33 \\
\hline 4 & FePcS-S & Acetato de etilo & 40 & 78 & 3 & 9 & 21 \\
\hline 5 & FePcS-S & $\mathrm{CH}_{3} \mathrm{CN}$ & 60 & 88 & 0 & 3 & 18 \\
\hline
\end{tabular}

$\mathrm{S}: \mathrm{SiO}_{2}, \mathrm{PE}$ : epóxido de a-pineno, $V$ : verbenol, $V E$ : verbenona. ${ }^{a}$ Condiciones de reacción: $4 \mathrm{~mL}$ de substrato en el solvente $0.125 \mathrm{M}$, $0.5 \%$ mol catalizador, $1.3 \mathrm{mmol}$ en $\mathrm{CH}_{2} \mathrm{Cl}_{2}(3.5 \mathrm{M}), 24 \mathrm{~h} .{ }^{b}$ Rendimiento $=($ mol de producto $) /($ mol inicial de $\alpha$-pineno $)$.

mayoritario con una selectividad de $21 \%$ (Lindmark-Henriksson et al., 2003).

\subsubsection{Sistemas catalíticos biomiméticos}

La oxidación de $\alpha$-pineno se ha realizado con las porfirinas $\mathrm{TPPFeCl}$ y $\mathrm{TMPFeCl}$ y $\mathrm{O}_{2}$ como oxidante (Hennig \& Luppa, 1999). Con el catalizador TPPFeCl los productos de epoxidación se obtienen con una selectividad de $23 \%$ y con $\mathrm{TMPFeCl}$ los únicos productos son verbenol y verbenona con selectividades de 68 y $32 \%$, respectivamente.

Con $\mu$-oxo-bis[2,9,16,23-tetrabutilftalocianato de Fe (III) y $\mu$-oxo-bis $[2,9,16,23$ tetrabutilftalocianato de $\mathrm{Mn}$ y $\mathrm{O}_{2}$ como oxidante (Weber et al. 1993), se obtuvieron selectividades similares $(25$ - $30 \%)$ al epóxido de $\alpha$-pineno, el verbenol y la verbenona. En estas reacciones, las especies ${ }^{\mathrm{t}} \mathrm{BuPcM}^{\mathrm{IV}}=\mathrm{O}$ inicialmente abstraen el hidrógeno alílico y posteriormente se recombinan con oxígeno molecular o con radicales hidroxilos derivados de las especies ${ }^{t} \mathrm{BuPcM}^{\mathrm{III}}-\mathrm{OH}$ formadas. El epóxido se forma principalmente por la transformación de especies peroxi-pineno a través del mecanismo de Schenck (Weber et al., 1993).

En la oxidación del $\alpha$-pineno con una $\mathrm{Cu}$ ftalocianina $\left(90{ }^{\circ} \mathrm{C}, \mathrm{O}_{2}\right)$ se obtuvieron selectividades de 40, 27 y $19 \%$, al epóxido, verbenol y verbenona, respectivamente (Rothenberg et al., 1998). La producción mayoritaria de epóxido se atribuyó a la estructura rígida del $\alpha$-pineno y a un solapamiento orbital que no permitió la eliminación del hidrógeno alílico.

La ftalocianina de hierro d-FePcS sin inmovilizar e inmovilizada en sílica se utilizó en la oxidación alílica de $\alpha$-pineno (Tabla 6) con TBHP (Pérollier \& Sorokin, 2002; González et al., 2004; González et al., 2005; González et al., 2006). La conversión del sustrato y la selectividad a la cetona fue mayor con $\alpha$-pineno que con limoneno como substrato (Tabla 3). La disminución en el rendimiento a verbenona al inmovilizar el complejo, se podría deber a impedimentos estéricos para acceder a los sitios activos del catalizador (entradas 1 y 2). Al aumentar la temperatura, disminuyó la conversión pero no se afectó el rendimiento a la verbenona debido a un aumento en la selectividad (entradas 2 y 5).

Al variar el solvente (entradas 2-4) se encontró que con acetona se obtiene el mayor rendimiento $(33 \%)$ y selectividad (44 \%) a la verbenona (entrada 3).

\section{Conclusiones}

Aunque existen varios sistemas catalíticos y no catalíticos reportados para la síntesis de carveol, carvona, verbenol y verbenona a partir de los monoterpenos limoneno y $\alpha$-pineno, en la mayoría de los casos no es posible conjugar altas conversiones y selectividades con condiciones no contaminantes. Aunque no existe aún un proceso 
técnica y económicamente viable para aplicaciones industriales, la demanda de estos productos y la tendencia a nivel mundial de implementar procesos más limpios, hacen necesario mantener los esfuerzos en este campo de investigación. Con los sistemas catalíticos basados en $\mathrm{Cr}$ se han obtenido las mayores actividades en la oxidación alílica de estos monoterpenos, pero presentan inconvenientes de lixiviación del metal lo que genera un problema ambiental por su toxicidad. Las ftalocianinas son materiales promisorios para la oxidación alílica de limoneno y $\alpha$-pineno. Además de hierro, es posible inmovilizar otros metales activos como $\mathrm{Cr}$ o $\mathrm{Pd}$ con $\mathrm{Cl}$ o $\mathrm{Br}$ como grupos sustituyentes. Como agente oxidante la combinación de oxígeno molecular con TBHP mejora tanto la conversión como la selectividad hacia los productos de oxidación alílica.

\section{Agradecimientos}

Los autores agradecen el apoyo financiero a la Universidad de Antioquia y a Colciencias mediante CENIVAM RC No. 432. R. de J. Barrera, E. A. Alarcón y L. M. González agradecen a Colciencias las becas para estudios doctorales.

\section{Referencias bibliográficas}

Agrawal, R., \& Joseph, R. (2000). Bioconversion of alpha pinene to verbenone by resting cells of Aspergillus niger. Applied Microbiology and Biotechnology. 53 (3), 335-337.

Allal, B.A., Firdoussi, L.E., Allaoud, S., Karim, A., Castanet, Y., \& Mortreux, A. (2003). Catalytic oxidation of $\alpha$-pinene by transition metal using t-butyl hydroperoxide and hydrogen peroxide. Journal of Molecular Catalysis A 200 (1-2), 177-184.

Arata, K., \& Tanabe, K. (1976). Isomerization of d-limonene oxide over aluminas. Chemistry Letters 5 (4), 321-322.

Booth, A.B., \& Klein, E.A. (1957). Preparation of carvone. United States Patent No. 2,796,428.
Bussi, J., López, A., Peña, F., Timbal, P., Paz, D., Lorenzo, D., \& Dellacasa, E. (2003). Liquid phase oxidation of limonene catalyzed by palladium supported on hydrotalcites. Applied Catalysis A $253(1), 177-189$.

Carey, F.A., \& Sundberg, R.A. (1990). Advanced organic chemistry. Third Edition, Plenum Press: New York.

Carloni, S., Frullanti, B., Maggi, R., Mazzacani, A., Bigi, F., \& Sartori, G. (2000). Allylic oxidation of olefins in the presence of Cu-Na-HSZ-320 zeolite as reusable solid catalyst. Tetrahedron Letters 41 (46), 8947-8950.

Carter, O.A., Peters, R.J., \& Croteau, R. (2003). Monoterpene biosynthesis pathway construction in Escherichia coli. Phytochemistry 64 (2), 425-433.

Casuscelli, S.G., Crivello, M.E., Perez, C.F., Ghione, G., Herrero, E.R., Pizzio, L.R., Vázquez, P.G., Cáceres, C.V., \& Blanco, M.N. (2004). Effect of reaction conditions on limonene epoxidation with $\mathrm{H}_{2} \mathrm{O}_{2}$ catalyzed by supported Keggin heteropolycompounds. Applied Catalysis A 274 (1-2), 115-122.

Catino, A.J., Forslund, R.E., \& Doyle, M.P. (2004). Dirhodium(II) caprolactamate: an exceptional catalyst for allylic oxidation. Journal of the American Chemical Society 126 (42), 13622-13623.

Choudary, B.M., Prasad, A.D., Swapna, V., Valli, V.L.K., \& Bhuma, V. (1992). Chromium pillared clay catalysed allylic oxidation and oxidative deprotection of allyl ethers and amines: a simple and convenient procedure. Tetrahedron 48 (5), 953-962.

Clark, J.S., Clarke, M.R., Clough, J., Blake, A.J., \& Wilson, C. (2004). Asymmetric allylic oxidation of bridge-bicyclic alkenes using a copper-catalysed symmetrising-desymmetrising Kharasch-Sosnovsky reaction. Tetrahedron Letters 45(51), 9447-9450. 
Coppen, J.J.W, \& Hone, G.A. (1995). Gum naval stores: turpentine and rosin from pine resin. Natural Resources Institute, Food and Agriculture Organization of the United Nations, Rome.

http://www.fao.org/docrep/v6460e/v6460e00.htm

Corvi-Mora, P., \& Ranise, A. (2003). (-)-Verbenone derivatives. United States Patent No. 6,649,658.

Crowell, P.L. (1997). Monoterpenes in breast cancer chemoprevention. Breast Cancer Research and Treatment 46 (2-3), 191-197.

Da Silva, M.J., Robles-Dutenhefner, P., Menini, L., \& Gusevskaya, E.V. (2003). Cobalt catalyzed autoxidation of monoterpenes in acetic acid and acetonitrile solutions. Journal of Molecular Catalysis A 201 (1-2), 71-77.

Dauben, W.G., Lorder, M., \& Fullerton, D.S. (1969). Allylic oxidation of olefins with chromium trioxide-pyridine complex. Journal of Organic Chemistry 34 (11), 3587-3592.

Davey, P.N., Newman, C.P., Thiam, W.A., \& Tse, C.L. (2002). Preparation of carvone. United States Patent No. 6,500,989.

De Carvalho, C.C.R., \& Da Fonseca, M.M.R. (2006a). Research review paper: biotransformations of terpenes. Biotechnology Advances 24 (2), 134-142.

De Carvalho, C.C.R., \& Da Fonseca, M.M.R. (2006b). Carvone: why and how should one bother to produce this terpene. Food Chemistry 95 (3), 413-422.

Department of Primary Industries (2002). Essential oils. Agriculture notes, State of Victoria, AG0656, p. 1-3.

Derfer, J.M., Kane, B.J., \& Young, D. G. (1966). Preparation of carvone. United States Patent No. 3,293,301.
Duetz, W.A., Witholt, B., \& Jourdat, C. (2000). Process for the preparation of trans-carveol. European Patent No. 1205556A1.

Dulou, R., Magnin, P., \& Bechtel, P. (1977). Process for the manufacture of a new derivative of alpha-pinene and new medicaments based on this derivative. United States Patent No. 4,036,884.

Eames, J., \& Watkinson, M. (2001). Catalytic Allylic oxidation of alkenes using an asymmetric Kharasch-Sosnovsky reaction. Angewandte Chemie (International Edition in English) 40 (19), 3567-3571.

Ernst, S., Gläser, R., \& Selle, M. (1997). MCM-41-type molecular sieves as carriers for metal-phthalocyanine complexes. In: H. Chon, S.-K. Ihm, Y.S. Uh (editors), Progress in zeolite and microporous materials. Studies in Surface Science and Catalysis 105, 1021-1028.

Fdil, N., Romane, A., Allaud, S., Karim, A., Castanet, A., \& Mortreux, A. (1996). Terpenic olefin epoxidation using metals acetylacetonates as catalysts. Journal of Molecular Catalysis A $108(1), 15-21$.

Fdil, N., Ait-Itto, M.Y., Ali, M.A., Karim, A., \& Daran, J.C. (2002). Epoxydation aerobique des terpenes naturels: etude de l'activité catalytique des nouveaux complexes $\mathrm{Ru}$-1,2,4-triazepines. Tetrahedron Letters 43(48), 8769-8771.

Firdoussi, L.E., Benharref, A., Allaoud, S., Karim, A., Castanet, Y., Mortreux, A., \& Petit, F. (1992). Acétoxylation et méthoxylation d'oléfines terpéniques catalysées par le palladium(II). Journal of Molecular Catalysis 72(2), L1-L5.

Firdoussi, L.E., Baqqa, A., Allaoud, S., Ait-Allal, B., Karim, A., Castanet, Y., \& Mortreux, A. (1998). Selective palladium-catalysed functionalization of limonene: synthetic and mechanistic aspects. Journal of Molecular Catalysis A 135(1), 11-22. 
Francis, M.J., Grant, P.K., Low, K.S., \& Weavers, R.T. (1976). Diterpene chemistry. Part VI: $\mathrm{SeO}_{2} / \mathrm{H}_{2} \mathrm{O}_{2}$ oxidations of exocyclic olefins. Tetrahedron 32(1), 95-101.

Ganschow, M., Wöhrle, D., \& Schulz-Ekloff, G. (1999). Incorporation of differently substituted phthalocyanines into the mesoporous molecular sieve Si-MCM-41. Journal of Porphyrins and Phthalocyanines 3 (4), 299-309.

Georgiy, B., Shul'pin, G.B., Nizova, G.V., Kozlov, Y.N., Gonzalez C.L., \& Güss-Fink, G.S. (2004). Hydrogen peroxide oxygenation of alkanes including methane and ethane catalyzed by iron complexes in acetonitrile. Advanced Synthesis and Catalysis. 346(2-3), 317-332.

Gomes, M.F.T., \& Antunes, O.A.C. (1997). Autoxidation of limonene, $\alpha$-pinene and $\beta$-pinene by dioxygen catalyzed by $\mathrm{Co}(\mathrm{OAc})_{2} /$ bromide. Journal of Molecular Catalysis A 121 (2-3), 145-155.

Gonçalves, J.A., \& Gusevskaya, E.V. (2004). Palladium catalyzed oxidation of monoterpenes: multistep electron transfer catalytic systems $\mathrm{Pd}(\mathrm{OAc})_{2} /$ benzoquinone/ $\mathrm{M}(\mathrm{OAc})_{2}(\mathrm{M}=\mathrm{Cu}, \mathrm{Co}$ or $\mathrm{Mn})$ for the allylic oxidation of limonene with dioxygen. Applied Catalysis A 258 (1), 93-98.

Gonçalves, J.A., Da Silva, M.J., Piló-Veloso, D., Howarth, O.W., \& Gusevskaya, E. V. (2005). Palladium catalyzed oxidation of monoterpenes: NMR study of palladium(II)-monoterpene interactions. Journal of Organometallic Chemistry 690 (12), 2996-3003.

Gonçalves, J.A., Bueno, A.C., \& Gusevskaya, E.V. (2006). Palladium-catalyzed oxidation of monoterpenes: highly selective syntheses of allylic ethers from limonene. Journal of Molecular Catalysis A 252 (1-2), 5-11.

González, L.M., Villa de P., A.L., Sorokin, A., \& Montes de C., C. (2004). Oxidación alílica de a-pineno con ftalocianinas de hierro inmovilizadas en silica. En Memorias del XIX Congreso Iberoamericano de Catálisis, Mérida, Yucatán, México, p. 2843-2850.
González, L.M., Villa de P., A.L., Sorokin, A., \& Montes de C., C. (2005). Allylic oxidation of monoterpenes over iron phthalocyanines immobilized on silica. In Proceedings of the 19th North American Catalysis Society Meeting, Philadelphia, Pennsylvania, USA.

González, L.M., Villa de P., A.L., Montes de C., C., \& Sorokin, A. (2006). Allylic oxidation of cyclohexene over silica immobilized iron tetrasulfophthalocyanine. Tetrahedron Letters $47(36), 6465-6468$.

González, L.M. (2007). Monoterpenes oxyfuncionalization. Tesis de doctorado, Posgrado en Ciencias Químicas, Universidad de Antioquia, Medellín, Colombia.

Gorzynski-Smith, J. (1984). Synthetically useful reactions of epoxides. Synthesis (8), 629-656.

Grootboom, N., \& Nyokong, T. (2002). Iron perchloroph ta loc y a n in e and tetrasulfophtalocyanine catalyzed oxidation of cyclohexane using hydrogen peroxide, chloroperoxybenzoic acid and tertbutylhydroperoxide as oxidants. Journal of Molecular Catalysis A 179(1-2), 113-123.

Gusevskaya, E., \& Gonçalves, J.A. (1997). Palladium(II) catalyzed oxidation of naturally occurring terpenes with dioxygen. Journal of Molecular Catalysis A 121(2-3), 131-137.

Harre, M., Haufe, R., Nickisch, K., Weinig, P., Weinmann, H., Kinney, W.A., \& Zhang, X. (1998). Some reaction safety aspects of ruthenium-catalyzed allylic oxidations of $\Delta$-5-steroids in the pilot plant. Organic Process Research \& Development 2 (2), 100-104.

Haudenschild, C., Schalk, M., Karp, F., \& Croteau, R. (2000). Functional expression of regiospecific cytochrome $\mathrm{P} 450$ limonene hydroxylases from mint (Mentha spp.) in Escherichia coli and Saccharomyces cerevisiae. Archives of Biochemistry and Biophysics 379 (1), 127-136. 
Hennig, H., \& Luppa, D.J. (1999). Photokatalytische Aktivierung von Oxygen by Iron(III) Porphyrins. Journal für Praktische Chemie 341(8), 757-767.

Holsten, E.H., Burnside, R.E., \& Seybold, S.J. (2000). Attractant semiochemicals of the engraver beetle, Ips perturbatus, in south-central and interior Alaska. United States Department of Agriculture, Forest Service, Pacific Northwest Research Station, Research Paper PNW-RP-529. http://www.entomology.umn.edu/Faculty/Seybol d/Holsten.pdf

Kala-Raj, N.K., Puranik, V.G., Gopinathan, C., \& Ramaswamy, A.V. (2003). Selective oxidation of limonene over sodium salt of cobalt containing s andwich-type polyoxotungstate $\left[\mathrm{WCo}_{3}\left(\mathrm{H}_{2} \mathrm{O}\right)_{2}\left(\mathrm{~W}_{9} \mathrm{CoO}_{34}\right)_{2}\right]^{10-}$. Applied Catalysis $A$ 256(1-2), 265-273.

Karandikar, P., Chandwadkar, A.J., Agashe, M., Ramgir, N.S., \& Sivasanker, S. (2006). Liquid phase oxidation of alkanes using $\mathrm{Cu} / \mathrm{Co}-$ perchlorophthalocyanine immobilized MCM-41 under mild reaction conditions. Applied Catalysis A 297 (2), 220-230.

Kolomeyer, G.G., \& Oyloe, J.S. (2004). Catalyst system and process for rearrangement of epoxides to allylic alcohols. United States Patent No. 6,835,686.

Kolomeyer, G.G., \& Oyloe, J.S. (2005). Method for preparation of alpha, beta-unsaturated cyclic ketones by dehydrogenation of secondary allylic cyclic alcohols. United States Patent No. 6,884,913.

Lajunen, M., \& Koskinen, A.M.P. (1994). Co(II)-catalysed allylic oxidation of $\alpha$-pinene by molecular oxygen; synthesis of verbenone. Tetrahedron Letters 35 (25), 4461-4464.

Lajunen, M.K. (2001). Co(II) catalysed oxidation of $\alpha$-pinene by molecular oxygen: Part III. Journal of Molecular Catalysis A 169 (1-2), 33-40.
Lajunen, M.K., Myllykoski, M., \& Asikkala, J. (2003). Co(II)-catalysed oxidation of $\alpha$-pinene by molecular oxygen: Part IV. Journal of Molecular Catalysis A 198 (1-2), 223-229.

Lempers, H.E.B., \& Sheldon, R.A. (1996). Allylic oxidation of olefins to the corresponding $\alpha, \beta$ unsaturated ketones catalyzed by chromium aluminophosphate-5. Applied Catalysis A 143 (1), 137-143.

Lindmark-Henriksson, H.M., Isaksson, D., Sjödin, K., Högberg, H.E., Vaněk, T., \& Valterová, I. (2003). Transformation of $\alpha$-pinene using a Picea abies suspension culture. Journal of Natural Products 66(3), 337-343.

MacTavish, H., \& Harris, D. (2002). An economic study of essential oil production in the UK: a case study comparing non-UK lavender / lavandin production and peppermint / spearmint production with UK production techniques and costs. ADAS Consulting Limited Report for the Government Industry Forum for Non-food Crops. MAFF, Food Surveillance Information Sheet No. 30 .

Maksimchuk, N.V., Melgunov, M.S., MrowiecBialon, J., Jarzebski, A.B., \& Kholdeeva, O.A. (2005). $\mathrm{H}_{2} \mathrm{O}_{2}$-based allylic oxidation of $\alpha$-pinene over different single site catalysts. Journal of Catalysis 235 (1), 175-183.

Marwah, P., \& Lardy, H.A. (1999). Process for effecting allylic oxidation. United States Patent No. $5,869,709$.

Marwah, P., \& Lardy, H.A. (2000). Process for effecting allylic oxidation. United States Patent No. 6,111,118.

Marwah, P., Lardy, H.A. (2002). Process for effecting allylic oxidation using dicarboxylic acid imides and chromium reagents. United States Patent No. 6,384,251.

Marwah, P., \& Lardy, H.A. (2004). Process for effecting allylic oxidation. United States Patent No. 6,686,486. 
McMorn, P., Roberts, G., \& Hutchings, G.J. (2000). Oxidation of $\alpha$-pinene to verbenone using silica-titania co-gel catalyst. Catalysis Letters 67 (2-4), 203-206.

Mikami, Y. (1988). Microbial conversion of terpenoids. In: G.E. Russell (editor), Biotechnology and Genetic Engineering Reviews. Intercept Scientific, Medical and Technical Publications, Wimborne, Dorset, United Kingdom, Vol. 6, p. 271-320.

Moiseev, I.I., \& Vargaftik, M.N. (2004). Allylic oxidation of alkenes with palladium catalysts. Coordination Chemistry Reviews 248 (21-24), 2381-2391.

Motherwell, W.B., Bingham, M.J., Pothier, J., \& Six, Y. (2004). A study of some molecularly imprinted polymers as protic catalysts for the isomerization of $\alpha$-pinene oxide to trans-carveol. Tetrahedron 60(14), 3231-3241.

Murphy, E.F., Mallat, T., \& Baiker, A. (2000). Allylic oxofunctionalization of cyclic olefins with homogeneous and heterogeneous catalysts. Catalysis Today 57 (1-2), 115-126.

Noma, Y., Yamasaki, S., \& Asakawa, Y. (1992). Biotransformation of limonene and related compounds by Aspergillus cellulosae. Phytochemistry 31 (8), 2725-2727.

Ohloff, G., \& Giersch, W.K. (1979). Process for the preparation of ipsdienol. United States Patent No. 4,157,451.

Oliveira, P., Ramos, A. M., Fonseca, I., Botelho do Rego, A., \& Vital, J. (2005). Oxidation of limonene over carbon anchored transition metal Schiff base complexes: effect of the linking agent. Catalysis Today 102 -103, 67-77.

Ooi, T., Miura, T., Itagaki, Y., Ichikawa, H., \& Maruoka, K. (2002). Catalytic MeerweinPonndorf-Verley (MPV) and Oppenauer (OPP) reactions: remarkable acceleration of the hydride transfer by powerful bidentate aluminum alkoxides. Synthesis (2), 279-291.
Ordonez, J., Smolcic, N., Weaver, M., \& Zhao, G. (2007). Synthesis of carvone from limonene. University of Michigan,Department of Chemistry. www.umich.edu/ chemh215/CHEM216/Honors Cup/hcproposal/231_D.pdf

Passaro, L.C., \& Webster, F.X. (2004). Synthesis of the female sex pheromone of the citrus Mealybug, Planococcus citri. Journal of Agricultural and Food Chemistry 52 (10), 2896-2899.

Pergentino de S., D., Ferreira de F.-N., \& Nobrega de A., R. (2007). Influence of the chirality of $(R)-(-)$ and $(S)-(+)$-carvone in the central nervous system: a comparative study. Chirality 19 (4), 264-268.

Pérollier, C., \& Sorokin, A.B. (2002). Preparation of $\alpha, \beta$-acetylenic ketones by catalytic heterogeneous oxidation of alkynes. Chemical Communications (14), 1548-1549.

Peterson, L., \& Bienvenu, F. (2007). Spearmint. http://www.rirdc.gov.au/pub/handbook/spearmin t.html

Popp, B.V., Thorman, J.L., \& Stahl, S.S. (2006). Similarities between the reactions of dioxygen and alkenes with palladium( $(0)$ : relevance to the use of benzoquinone and molecular oxygen as stoichiometric oxidants in palladium-catalyzed oxidation reactions. Journal of Molecular Catalysis A 251 (1-2), 2-7.

Quiroz, N., Stashenko, E., Paez, E.A., \& Martínez, J.R. (1999). Zeolitas NaY intercambiadas con metales de transición $\left(\mathrm{Fe}^{2+}, \mathrm{Co}^{2+}, \mathrm{Mo}^{2+}, \mathrm{y} \mathrm{Mn}^{2+}\right)$ como catalizadores para la oxidación de limoneno. Revista Colombiana de Química 28 (1), 45-53.

Ravid, U., Putievsky, E., Katzir, I., Lewinsohn, E., \& Dudai, N. (1997). Identification of $(1 R)(+)$-verbenone in essential oils of Rosmarinus officinalis L. Flavour and Fragrance Journal $12(2), 109-112$. 
Reddy, M. M., Punniyamurthy, T., \& Iqbal, J. (1995). Cobalt catalyzed oxidation of cyclic alkenes with molecular oxygen: allylic oxidation versus double bond attack. Tetrahedron Letters $36(1), 159-162$.

Rhodes, P.M., \& Winskill, N. (1985). Microbiological process for the preparation of 1- carvone. United States Patent No. 4,495,284.

Robles-Dutenhefner, P.A., Nunez, D.L., Gonçalves, J.A., Gusevskaya, E.V., \& Sousa, E.M.B. (2004a). Sol-gel palladium composites: effect of the thermal treatment on the catalytic activity. Journal of Non-Crystalline Solids 348, 195-200.

Robles-Dutenhefner, P.A., Da Silva, M.J., Sales, L.S., Sousa, E.M.B., \& Gusevskaya, E.V. (2004b). Solvent-free liquid-phase autoxidation of monoterpenes catalyzed by sol-gel $\mathrm{Co} / \mathrm{SiO}_{2}$. Journal of Molecular Catalysis A 217 (1-2), 139-144.

Rothenberg, G., Yatziv, Y., \& Sasson, Y. (1998). Comparative autoxidation of 3-carene and $\alpha$-pinene: factors governing regioselective hydrogen abstraction reactions. Tetrahedron 54 (3-4), 593-598.

Safari, N., \& Bahadoran, F. (2001). Cytochrome P-450 model reactions: a kinetic study of epoxidation of alkenes by iron phthalocyanine. Journal of Molecular Catalysis A 171 (1-2), 115-121.

Sakthivel, A., Dapurkar, S.E., \& Selvam, P. (2003). Allylic oxidation of cyclohexene over chromium containing mesoporous molecular sieves. Applied Catalysis A 246(2), 283-293.

Salavati-Niassary, M., Farzaneh, F., Ghandi, M., \& Turkian, L. (2000). Oxidation of cyclohexene with tert-butylhydroperoxide catalyzed by manganese(II) complexes included in zeolite Y. Journal of Molecular Catalysis A 157 (1-2), 183-188.
Salvador, J.A.R., \& Silvestre, S.M. (2005). Bismuth-catalyzed allylic oxidation using t-butyl hydroperoxide. Tetrahedron Letters 46 (15), 2581-2584.

Savithiry, N., \& Oriel, P.J. (1998). Method for production of monoterpene derivatives of limonene. United States Patent No. 5,763,237.

Sheldon, R.A., \& Kochi, J. (1981). Metal-catalyzed oxidations of organic compounds. New York: Academic Press.

Sheldon, R.A., Arends, I.W.C.E., \& Lempers, H.E.B. (1997). Heterogeneous catalysts for liquid phase oxidations. In: B.K. Hodnett, A.P. Kybett, J.H. Clark, \& K. Smith (editors), Supported reagents and catalysts in chemistry. The Royal Society of Chemistry, United Kingdom, p. 37-46.

Sheldon, R.A., Arends, I.W.C.E., \& Lempers, H.E.B. (1998). Liquid phase oxidation at metal ions and complexes in constrained environments. Catalysis Today 41 (4), 387-407.

Silva, A.D., Patitucci, M., Bizzo, H.R., D'Elia, E., \& Antunes, O.A.C. (2002). Wacker $\mathrm{PdCl}_{2}-\mathrm{CuCl}_{2}$ catalytic oxidation process: oxidation of limonene. Catalysis Communications 3 (9), 435-440.

Simakova, I.L., \& Semikolenov, V.A. (2003). The catalytic method of verbenol preparation with controlled isomer distribution starting from renewable material $\alpha$-pinene. Chemistry for Sustainable Development 11,271-275.

Singh, J., Sharma, M., Kad, G.L., \& Chhabra, B.R. (1997). Selective oxidation of allylic methyl groups over a solid support under microwave irradiation. Journal of Chemical Research (S), 264-265.

Slotnick, B., \& Bisulco, S. (2003). Detection and discrimination of carvone enantiomers in rats with olfactory bulb lesions. Neuroscience 121 (2), 451-457. 
Sorokin, A.B., \& Tuel, A. (1999). Heterogeneous oxidation of aromatic compounds catalyzed by metallophthalocyanine functionalized silicas. New Journal of Chemistry 23 (5), 473-476.

Sorokin, A.B., \& Tuel, A. (2000). Metallophthalocyanine functionalized silicas: catalysts for the selective oxidation of aromatic compounds. Catalysis Today 57 (1-2), 45-59.

Sorokin, A.B., Mangematin, S., \& Pergrale, C. (2002). Selective oxidation of aromatic compounds with dioxygen and peroxides catalyzed by phthalocyanine supported catalysts. Journal of Molecular Catalysis A 182-183, 267-281.

Stashenko, E.E., Jaramillo, B.E., \& Martínez, J.R. (2004). Comparison of different extraction methods for the analysis of volatile secondary metabolites of Lippia alba (Mill.) N.E. Brown, grown in Colombia, and evaluation of its in vitro antioxidant activity. Journal of Chromatography A 1025(1), 93-103.

Stultz, L.K., Huynh, M.H.V., Binstead, R.A., Curry, M., \& Meyer, T.J. (2000). Allylic oxidation of cyclohexene and indene by cis-[RuIV(bpy $\left.)_{2}(\mathrm{py})(\mathrm{O})\right]^{2+}$. Journal of the American Chemical Society 122 (25), 5984-5996.

Surferchicks.com. (2007). Perfume allergies. http://www.surferchicks.com/perfume.htm

Tachibana, S., Maegawa, Y., \& Nomura, M. (2007). Synthesis and physiological activity of ureas and amides with carvone residues. Journal of Oleo Science 56(6), 303-307.

Trachtenberg, E.N., Nelson, C.H., \& Carver, J.R. (1970). Mechanism of selenium dioxide oxidation of olefins. The Journal of Organic Chemistry $35(5), 1653-1658$.

Trissa, J., Sawant, D.P., Gopinath, C.S., \& Halligudi, S.B. (2002). Zeolite encapsulated ruthenium and cobalt Schiff base complexes catalyzed allylic oxidation of $\alpha$-pinene. Journal of Molecular Catalysis A 184 (1-2), 289-299.
Trissa, J., \& Halligudi, S.B. (2005). Oxyfunctionalization of limonene using vanadium complex anchored on functionalized SBA-15. Journal of Molecular Catalysis A $229(1-2), 241-247$.

Umbreit, M.A., \& Sharpless, K.B. (1977). Allylic oxidation of olefins by catalytic and stoichiometric selenium dioxide with tert-butyl hydroperoxide. Journal of the American Chemical Society 99(16), 5526-5528.

Wang, R.-M., Hao, C.-J., Wang, Y.-P., \& Li, S.-B. (1999). Amino acid Schiff base complex catalyst for effective oxidation of olefins with molecular oxygen. Journal of Molecular Catalysis A $147(1-2), 173-178$.

Warshaw, E.M., \& Zug, K.A. (1996). Sesquiterpene lactone allergy. American Journal of Contact Dermatitis 7 (1), 1-23.

Weber, L., Grosche, M., Hennig, H., \& Haufe, G. (1993). Oxygenation of alkenes with phthalocyaninato manganese (III) and iron (III) complexes and dioxygen. Journal of Molecular Catalysis 78(1), L9-L14.

Wender, P.A., \& Mucciaro, T.P. (1992). A new and practical approach to the synthesis of taxol and taxol analogues: the pinene path. Journal of the American Chemical Society 114(14), 5878-5879.

Zefirov, N.S., \& Zakharov, A.N. (1998). Catalysis by topologically anchored metal complexes. Liquid-phase hydroxylation of benzene by $\mathrm{H}_{2} \mathrm{O}_{2}$ in the presence of zeolite-supported $\mathrm{Fe}$ (II) phthalocyanine. Canadian Journal of Chemistry $76(6), 955-959$. 